\title{
Adrenal haemorrhage in term neonates: a retrospective study from the period 2001-2013
}

\section{Zita Gyurkovits, Ágnes Maróti, Lóránd Rénes, Gábor Németh, Attila Pál \& Hajnalka Orvos}

To cite this article: Zita Gyurkovits, Ágnes Maróti, Lóránd Rénes, Gábor Németh, Attila Pál \& Hajnalka Orvos (2015) Adrenal haemorrhage in term neonates: a retrospective study from the period 2001-2013, The Journal of Maternal-Fetal \& Neonatal Medicine, 28:17, 2062-2065, DOI: 10.3109/14767058.2014.976550

To link to this article: https://doi.org/10.3109/14767058.2014.976550

Accepted author version posted online: 20

Oct 2014.

Published online: 07 Nov 2014.

\section{Submit your article to this journal $c$}

Џ Article views: 260

Q View related articles $\sqsubset$

View Crossmark data [ᄌ

4 Citing articles: 1 View citing articles 


\title{
Adrenal haemorrhage in term neonates: a retrospective study from the period 2001-2013
}

\author{
Zita Gyurkovits ${ }^{1}$, Ágnes Maróti ${ }^{2}$, Lóránd Rénes ${ }^{1}$, Gábor Németh ${ }^{1}$, Attila Pál ${ }^{1}$, and Hajnalka Orvos ${ }^{1}$ \\ ${ }^{7}$ Department of Obstetrics and Gynaecology and ${ }^{2}$ Department of Paediatrics, University of Szeged, Szeged, Hungary
}

\begin{abstract}
Objective: To assess the incidence, risk factors and clinical presentations of neonatal adrenal haemorrhage (NAH) in uncomplicated, singleton and term deliveries.

Methods: A retrospective analysis of 26416 term neonates delivered between 2001 and 2013, and screened with abdominal ultrasonography.

Results: Of the 26416 neonates, $74(0.28 \%)$ displayed NAH; the male/female ratio was 1.55:1. Vaginal delivery was significantly more frequent than caesarean section among them ( 71 versus 3; $95.9 \%$ versus $4.1 \%)$. Unilateral bleeding occurred on the right side in 36 (48.7\%), and on the left in $34(45.9 \%)$, without a significant difference; bilateral haematomas were found in four cases $(5.4 \%)$. The most common risk factors were macrosomia $(16,21.6 \%)$ and fetal acidaemia $(23,31 \%)$, while four $(5.4 \%)$ neonates exhibited pathological acidaemia. Clinical presentations included jaundice in $37(50 \%)$, anaemia in six (8.1\%) and an adrenal insufficiency in only one $(1.3 \%)$ case. In three cases, neuroblastoma was diagnosed.

Conclusions: Vaginal delivery, macrosomia and fetal acidaemia are the most important risk factors for $\mathrm{NAH}$. The adrenal glands on both sides were similarly involved. In the healthy neonates with $\mathrm{NAH}$, the clinical presentations were mild, with spontaneous regression. Differentiation of NAH from tumours is of considerable importance.
\end{abstract}

Keywords

Adrenal haemorrhage, neonate, ultrasonographic screening

\author{
History \\ Received 20 July 2014 \\ Revised 14 September 2014 \\ Accepted 10 October 2014 \\ Published online 7 November 2014
}

\section{Introduction}

Adrenal bleeding in the perinatal period may remain asymptomatic and, as routine neonatal abdominal screening is not common practice worldwide, its exact incidence cannot be determined. Earlier estimates indicated $1.7-2.1$ per 1000 births, depending on the mode of diagnosis, which in most cases is ultrasonographic (USG) screening [1]. The pathogenesis is still unclear; the extensive vascularity and the relatively large size enhance the vulnerability of the adrenal glands to venous pressure changes, and mechanical compression also has a role during delivery [2]. Predisposing factors include prematurity, prolonged labour, a difficult delivery, macrosomia, perinatal hypoxia, coagulation disorders and septicaemia, though in most cases the aetiology cannot be established [3]. Neonatal adrenal haemorrhage (NAH) can occur in utero, but it usually appears perinatally; $5-10 \%$ of the cases involve bilateral haemorrhage; interestingly, in the unilateral cases, right adrenal gland involvement has been reported more frequently [4]. The clinical features are variable: anaemia, persistent indirect hyperbilirubinaemia, abdominal distension with an abdominal mass, and a bluish

Address for correspondence: Zita Gyurkovits, Department of Obstetrics and Gynaecology, University of Szeged, Semmelweis Street 1, 6725 Szeged, Hungary. Tel: +36 70 4083878. Fax: +36 62 545711. E-mail: gyurkovits2000@yahoo.com discolouration of the scrotum [5]. Whereas minor bleeding into the adrenal cortex may remain asymptomatic, a severe blood loss can be life-threatening, with hypovolaemic shock or an adrenal insufficiency. The differential diagnosis of suprarenal masses should include neuroblastoma, the predominant neonatal malignancy, teratomas, subdiaphragmatic extralobar pulmonary sequestration, vascular thrombosis and congenital adrenal cystic lesions [6].

In this study, we set out to review the incidence, risk factors and clinical presentations of NAH in term infants in our neonatal department over a 13-year period.

\section{Methods}

This was a retrospective study on singleton pregnancies of women who delivered at the Department of Obstetrics and Gynaecology, University of Szeged, Hungary, between 01.01.2001 and 31.12.2013. The inclusion criteria included a gestational age at delivery of at least 37 completed weeks; 26416 full-term mature neonates of either sex, born between gestational weeks 37 and 41, were enrolled. Neonates who needed neonatal intensive care immediately after birth were not included in the study. The maternal data, gestational age, parity, mode of delivery and the presence of gestational diabetes mellitus were reviewed.

Every baby had ultrasound examination on day 2 of their life; this practice was adapted to screen renal abnormalities. 
Considering that our university is a tertier center with a numerous transfers from other regions without proper antenatal care, our department decided to introduce renal screening. The significance of renal screening is wellknown in the literature, where sonographic screening of the kidneys and the urinary tract was described as a very effective and non-invasive screening method after birth. It allows planning for appropriate diagnostic tests and therapeutic procedures in a timely fashion [7-9].

Suprarenal masses were diagnosed via postnatal USG of the abdomen on the second day of postnatal life, and the site of adrenal haemorrhage was recorded.

From the data in the files on the neonates, we analysed the presence of risk factors and comorbidities, such as a high birthweight, fracture of the clavicle, cephalhaematoma, anaemia, hyperbilirubinaemia, hypoglycaemia and respiratory disorders. The neonatal outcome was investigated from the aspects of the umbilical cord blood $\mathrm{pH}$, the base excess (BE) and the 5-10 min Apgar scores. The neonatal outcomes were compared between two groups: the neonates with NAH and the control group, 5167 consecutive healthy term neonates born in a 2-year period (2012 and 2013). We found that this approach is statistically proper and allows a sufficiently correct and relevant basis of reference as a control group for comparison with the NAH group.

Immediately after delivery, a segment of the umbilical cord was double-clamped, and blood was drawn from the artery into preheparinized plastic syringes. Coagulation was inhibited with EDTA. The whole-blood samples were analysed within $5 \mathrm{~min}$ of collection for $\mathrm{pH}$ and BE. Acidaemia was defined as an umbilical blood $\mathrm{pH}<7.2$ or/and an umbilical $\mathrm{BE}<-12 \mathrm{mmol} / \mathrm{l}$ (a BE of $-12 \mathrm{mmol} / 1$ is approximately 2SDs below the mean), and pathological fetal acidaemia was diagnosed when the umbilical blood $\mathrm{pH}$ was $<7.0$. Maternal acidaemia as a cause of the cord blood acidaemia was excluded.

Macrosomia was defined as a birthweight $>4000 \mathrm{~g}$, and anaemia as a haemoglobin level more than 2SDs below the mean value for the corresponding age [10]. The definition of hypoglycaemia was a blood glucose level $<2.6 \mathrm{mmol} / 1$. Hyperbilirubinaemia was defined according to the Clinical Practice Guideline of the American Academy of Paediatrics published in 2004 [11].

Statistical analysis was performed by using the chi-square test; a level $p<0.05$ was considered to be statistically significant.

\section{Results}

Abdominal USG was applied in the total of 26416 singleton neonates included in the study from the 13-year period; 74 of the neonates demonstrated NAH, an incidence of $0.28 \%$. The vast majority, $71(95.9 \%)$ of these 74 neonates, were delivered by vaginal delivery, and only $3(4.1 \%)$ by caesarean section; the frequency of caesarean section in the $\mathrm{NAH}$ group was significantly lower than that in the control group $(40.6 \%)$.

The haemorrhagic lesions were evaluated by USG as inhomogeneous lesions with decreased echogenicity that were mixed solid-liquid or echogenic masses.
$\mathrm{NAH}$ was more frequent in boys $(60.8 \%)$ than in girls (39.2\%); the difference was significant. Thirty six neonates (48.7\%) had a unilateral haematoma on the right side, while $34(45.9 \%)$ had one on the left, with no significant difference between the sides. Bilateral haematomas were found in four cases $(5.4 \%)$.

The mean birthweight and gestational age were $3640 \pm 424 \mathrm{~g}$ and $39.1 \pm 1.0$ weeks, respectively; $29(39 \%)$ of the 74 were primipara and $45(61 \%)$ were multipara.

Sixteen $(21.6 \%)$ of the 74 neonates were macrosomic, i.e. a significantly higher proportion as compared with the macrosomic neonate rate $(7.1 \%)$ for the entire population at our department. Four mothers (5.4\%) were diagnosed with gestational diabetes mellitus, a similar level of incidence as in our previous data.

As concerns the general condition at birth of the neonates with $\mathrm{NAH}$, in $23(31 \%)$ the umbilical cord blood $\mathrm{pH}$ was $<7.2$, or/and had $\mathrm{BE}<-12 \mathrm{mmol} / \mathrm{l}$; fetal acidaemia was therefore significantly more frequent in the NAH group. Four $(5.4 \%)$ of the 23 displayed pathological acidaemia with an umbilical cord blood $\mathrm{pH}<7.0$. Five neonates $(6.7 \%)$ had a 5- or 10-min Apgar score $<7 \quad(p<0.05)$.

The most common significant clinical features in the neonates with NAH were indirect hyperbilirubinaemia, in 37 $(50.0 \%)$ cases, and anaemia in six $(8.1 \%)$ cases $(p<0.001)$. Other clinical presentations included birth trauma, e.g. cephalhaematoma $(5 ; 6.7 \%)$; as compared with the controls, this was very close to the limit of significance. In cases of clavicle fracture $(1 ; 1.3 \%)$ and hypoglycaemia $(2 ; 2.7 \%)$, significance calculations could not be carried out, because of the low incidence, but both clinical presentations were observed with similar incidence in the NAH and control groups.

We have not found any abnormal palpable masses among these 74 babies although all the newborns were examined at least two times by specialised neonatologists. There was no significant difference in the rates of respiratory disorders between the NAH and control group (8.1\% versus $6.3 \%$ ).

Table 1 summarises the statistical data and an analysis of the NAH and control groups. No perinatal mortality occurred in either study group.

One neonate (1.3\%) with bilateral NAH developed an adrenal insufficiency and was treated with glucocorticoid. Coagulation disorder and sepsis were not observed. The mean time for complete resolution of the haemorrhage was $18 \pm 8$ weeks. Three of the suprarenal masses proved to be congenital neuroblastomas (as confirmed by colour Doppler USG and pathological analysis) developing from the adrenal glands; these neonates were transferred and treated in another unit.

\section{Discussion}

This is the largest retrospective study that we are aware of which reports on the prevalence of NAH among term neonates. During a 13-year period, 74 neonates were diagnosed with NAH by abdominal USG, reflecting an incidence of $0.28 \%$ among the healthy term infants. Previous studies have indicated a wide range of incidence, from $0.003 \%$ up to $0.55 \%$, depending on whether the selected population was symptom-free or treated in an intensive care unit $[12,13]$ 
Table 1. Outcome measures of neonates with NAH and the control group.

\begin{tabular}{lccc}
\hline Comorbidities & NAH $(n=74)$ & Control $(n=5167)$ & $p$ \\
\hline Males & $45(60.8 \%)$ & $2602(50.3 \%)$ & $<0.05^{*}$ \\
Caesarean section & $3(4.1 \%)$ & $2100(40.6 \%)$ & $<0.001^{*}$ \\
Vaginal delivery & $71(95.9 \%)$ & $2955(57.2 \%)$ & $<0.001^{*}$ \\
Umbilical cord pH $<7.2$ & $23(31 \%)$ & $775(15 \%)$ & $<0.001^{*}$ \\
Apgar score $<7$ at 5 min & $5(6.7 \%)$ & $93(1.8 \%)$ & $<0.05^{*}$ \\
Macrosomia & $16(21.6 \%)$ & $366(7.1 \%)$ & $<0.001^{*}$ \\
Anaemia & $6(8.1 \%)$ & $87(1.7 \%)$ & $<0.001^{*}$ \\
Indirect hyperbilirubinaemia & $37(50 \%)$ & $1522(29.4 \%)$ & $<0.001^{*}$ \\
Cephalhaematoma & $5(6.7 \%)$ & $150(2.9 \%)$ & 0.052 \\
Clavicle fracture & $1(1.3 \%)$ & $50(1.0 \%)$ & - \\
Hypoglycaemia & $2(2.7 \%)$ & $163(3.1 \%)$ & - \\
Respiratory disorder & $6(8.1 \%)$ & $326(6.3 \%)$ & 0.52 \\
\hline
\end{tabular}

Significance at $p<0.05$ is indicated by*.

and also on whether the mode of diagnosis was USG, CT or autopsy.

Most of the neonates with NAH in our study were delivered by vaginal delivery, which is consistent with earlier findings and reflects the possible role of mechanical compression and also a hypoxic-ischaemic event with subsequent reperfusion injury during vaginal delivery [14]. Although the precise mechanisms leading to adrenal haemorrhage are still unclear, the available evidences have implicated the role of adrenocorticotropic hormone $(\mathrm{ACTH})$, adrenal vein spasm and thrombosis, the limited venous drainage in the pathogenesis of this condition. The adrenal gland has a rich arterial supply, in contrast to its limited venous drainage, which is critically dependent on a single vein. Furthermore, in stressful situations, ACTH secretion increases, which stimulates adrenal arterial blood flow and cause adrenal vein spasm so the limited venous drainage capacity may cause venous stasis and may lead to haemorrhage [10].

The occurrence of NAH was particularly high in the macrosomic group and in those with fetal acidaemia or birth asphyxia, which is again consistent with other reports in the literature [15]. Furthermore, the common clinical presentations were similar to those in previous reviews, i.e. anaemia and persistent indirect bilirubinaemia [16].

On the other hand, our study revealed that the right and left sides were equally likely to be involved, in contrast with former reports suggesting that the probability of right adrenal gland haemorrhage involvement was higher. The mechanism for a right-sided predilection is presumed to be the compression of the adrenal gland between the liver and the spine and the pressure fluctuation in the inferior vena cava, which is directly connected to the right adrenal gland. However, at our department each infant undergoes abdominal USG screening. This therefore furnished reliable data on a high number of neonates, clearly demonstrating the equal likelihood of left and right NAH.

It is noteworthy that the incidence of NAH exhibited a slightly increasing tendency during this 13-year period, despite the continuously falling rate of vaginal delivery in our department.

This phenomenon may be explained in part by the rising frequency of macrosomic neonates. During the last 2-3 decades an overall increase in the proportion of macrosomic newborns has been found. The causes of the increasing prevalence of large newborns are complex and insufficiently explained, however this is a common phenomenon in many developed countries. The incidence of macrosomia varies between $5 \%$ and $20 \%$, the highest ratio is found in the Nordic countries [17]. In Hungary, the incidences of macrosomia (birthweight $>4000 \mathrm{~g}$ ) in the recent decades are shown in Figure 1.

A number of risk factors for fetal macrosomia have been recognised, the strongest is the gestational maternal diabetes. Other modifiable risk factors are maternal pre-gestational anthropometric characteristics, like BMI, excessive weight gain during pregnancy, nutritional intake, level of physical activity, smoking and metabolic parameters. Non-modifiable factors include genes, fetal sex, parity, maternal age and height $[18,19]$.

In the event of a suprarenal mass, it is of the highest importance to differentiate bleeding from a malignancy, such as a neuroblastoma, which originates from the neural tube cells. The tumour can be differentiated by the presence of vascular flow with colour Doppler USG or CT and via the catecholamine metabolite levels in the urine in the case of a neuroblastoma. While conservative therapy is appropriate for $\mathrm{NAH}$, adrenal tumours may need surgical excision. An adrenal mass can be followed up for one month with serial USG without any adverse effects on the therapy and prognosis of the tumour [20], persistence or enlargement suggesting an adrenal neoplasm. We diagnosed three cases of neuroblastoma; at the time of detection, all of them were symptom-free, the early diagnosis therefore was promoting a good prognosis.

$\mathrm{NAH}$, and particularly the bilateral form, needs a careful follow-up to prevent the later consequences, including prolonged jaundice, anaemia or an adrenal insufficiency. In our study, jaundice was observed in 50\% and anaemia in $8.1 \%$ of the NAH cases; they required only conservative treatment. An adrenal insufficiency was diagnosed in only one patient, who needed adequate hormone supplementation.

The limitation of our study is its retrospective nature, which is why the exact descriptions of haemorrhages were not recorded. Although an adrenal insufficiency is more frequent among preterms than among mature neonates [21], preterms were not included in the study as they were transferred to 
Figure 1. Incidence of macrosomia (birthweight $>4000 \mathrm{~g}$ ) in Hungary from 1970 until 2010.

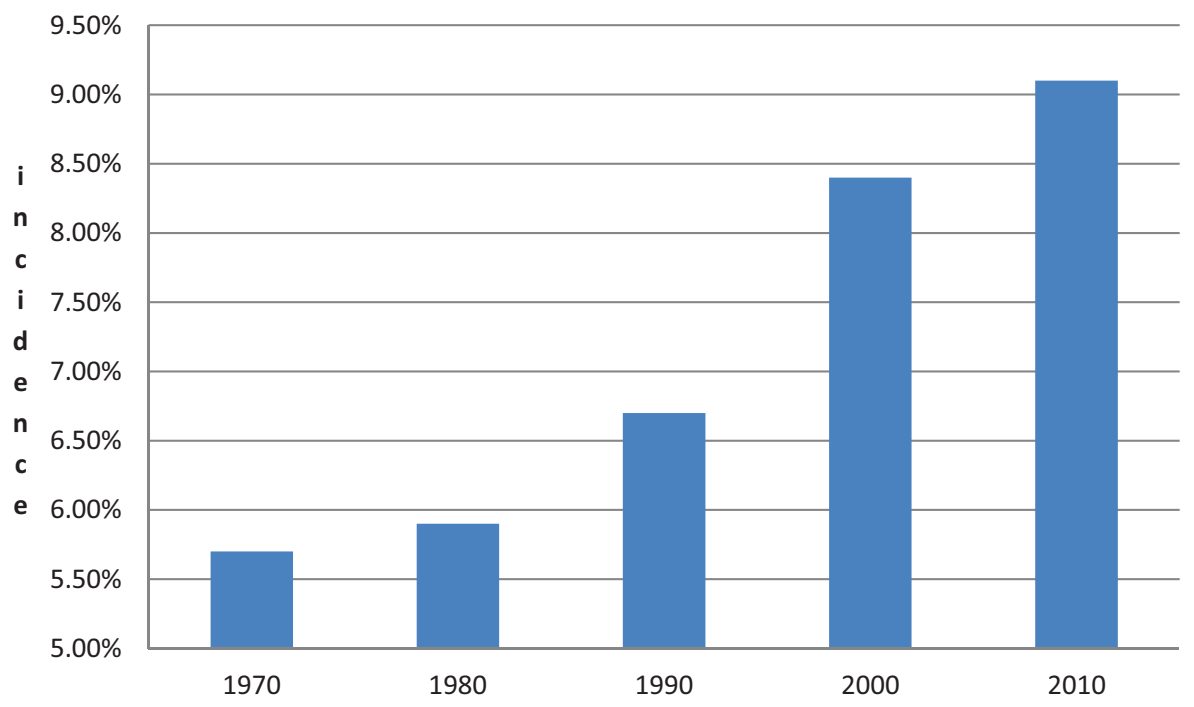

another department. Due to the same reason, the medical follow-up of the neonates with an adrenal insufficiency or neuroblastoma was not feasible.

Primary coagulation disorders and sepsis are also wellknown risk factors for bleeding, but they did not play a role in the aetiology in our work.

In conclusion, this is the largest series of scanning for NAH in healthy neonates that has been reported so far. The findings highlight the diverse and non-specific clinical presentation of NAH, though most of the cases had merely mild consequences and needed only conservative treatment.

We still continue our screening protocol regarding abdominal ultrasound screening for every term neonate, as benefits overweigh the harms, according to our opinion. Particularly, the follow-up of the newborns with any congenital abnormalities became much more effective and precise with this protocol. However, as further data and investigations are still needed to clarify the presence and importance of the later consequences, for other neonatal units we only recommend abdominal ultrasound screening in the risk groups, like macrosomic neonates with vaginal delivery or fetal acidaemia. A long term follow-up for the consequences of adrenal haemorrhage is still needed. The importance of abdominal USG screening is to be stressed, especially for vaginally delivered macrosomic neonates, for those with fetal acidaemia and for newborns suffering from birth trauma, unexplained anaemia and prolonged icterus.

\section{Declaration of interest}

The authors report no conflicts of interest.

\section{References}

1. Demirel N, Baş AY, Zenciroğlu A, et al. Adrenal bleeding in neonates: report of 37 cases. Turk J Pediatr 2011;53:43-7.

2. Felc Z. Ultrasound in screening for neonatal adrenal hemorrhage. Am J Perinatol 1995;12:363-6.

3. Katar S, Oztürkmen-Akay H, Devecioğlu C, et al. A rare cause of hyperbilirubinemia in a newborn: bilateral adrenal hematoma. Turk J Pediatr 2008;50:485-7.
4. Lai LJ, Chen LM, Chu PY, et al. Neonatal adrenal hemorrhage associated with scrotal hematoma: an unusual case report and literature review. Pediatr Neonatol 2012;53:210-12.

5. Velaphi SC, Perlman M. Neonatal adrenal hemorrhage: clinical and abdominal sonographic findings. Clin Pediatr 2001;40:545-8.

6. Postek G, Streich H, Narębski K. Assessment of diagnostic methods in adrenal gland hemorrhage in neonates on the basis of own material from the years 2007-2011. Pol J Radiol 2011;76:62-4.

7. Bereczki CS, Orvos H, Sumegi V, et al. Postnatal renal pelvis dilatation - results of 9650 screened neonates (Abstr). Pediatr Nephrol 2004;19:C217.

8. Gruessner SE, Klein K, Peter C, et al. Ultrasound screening of the kidneys and urinary tract in 11.887 newborn infants: a 10-year experience. Open J Obstet Gynecol 2012;2:389-93.

9. Drnasin K, Saraga-Babić M, Saraga M. Clinical importance of pyelocalyceal dilation diagnosed by postnatal of the ultrasonographic screening urinary tract. Med Sci Monit. 2013;19:125-31.

10. Tulassay T, Seri I, Evans J. Renal vascular disease in the newborn. In: Taeugah HW, Ballard RA, Avery ME, eds. Schaffers and avery's diseases of newborn. 7th ed. Philadelphia: WB Saunders Company; 1998:1177-87.

11. American Academy of Pediatrics. Clinical practice guideline. Management of hyperbilirubinemia in the newborn infant 35 or more weeks of gestation. Pediatrics 2004;114:297-308.

12. White PC. Adrenocortical insufficiency. In: Behrman RE, Kliegman RM, Jenson HB, eds. Nelson text book of pediatrics. 18th ed. Philadelphia, PA: Saunders; 2007:2357-8.

13. Lee MC, Lin LH. Ultrasound screening of neonatal adrenal hemorrhage. Acta Paediatr Taiwan 2000;41:327-30.

14. DeSa DJ, Nicholls S. Haemorrhagic necrosis of the adrenal gland in perinatal infants: a clinico-pathological study. J Pathol 1972;106: 133-49.

15. Örün E, Yıldırım M, Yılmaz AE, et al. Is routine abdominal ultrasonography necessary in macrosomic newborns with difficult delivery? Matern Fetal Neonatal Med 2012;25:1195-6.

16. Qureshi UA, Ahmad N, Rasool A, Choh S. Neonatal adrenal hemorrhage presenting as late onset neonatal jaundice. J Indian Assoc Pediatr Surg 2009;14:221-3.

17. Henriksen T. The macrosomic fetus: a challenge in current obstetrics. Acta Obstet Gynecol Scand 2008;87:134-45.

18. Kramer MS, Morin I, Yang H, et al. Why are babies getting bigger? Temporal trends in fetal growth and its determinants. J Pediatr 2002;141:538-42.

19. Rooth G. Increase in birthweight: a unique biological event and an obstetrical problem. Eur J Obstet Gynecol Reprod Biol 2003;106: 86-7.

20. Yao W, Li K, Xiao X, et al. Neonatal suprarenal mass: differential diagnosis and treatment. J Cancer Res Clin Oncol 2013;139:281-6.

21. Mutlu M, Karagüzel G, Aslan Y, et al. Adrenal hemorrhage in newborns: a retrospective study. World J Pediatr 2011;7:355-7. 